\title{
El derecho al consentimiento informado de las personas mayores en el ámbito de salud. Estándares desde el derecho internacional de los derechos humanos y sus efectos en el ordenamiento jurídico chileno
}

The Right to Informed Consent of Elderly Persons in Health. Standards from
the International Human Rights Law and its effects in Chilean Legal System

Edison CALAHORRANO LATORRE $\mathbf{1}$

Resumen: El presente trabajo analiza los estándares del derecho al consentimiento informado de las personas mayores en el ámbito sanitario. Se parte desde la necesidad de especialización en la protección de los derechos de las personas mayores. Luego, se analiza la innovación introducida por la Convención Interamericana para la Protección de los Derechos Humanos de las Personas Mayores respecto al consentimiento informado, su tratamiento en el Derecho Comparado y la jurisprudencia. Finalmente se analizan las obligaciones establecidas por la Corte Interamericana de Derechos Humanos en el caso Poblete Vilches vs. Chile y la auto ejecutabilidad de la Convención.

Palabras clave: Personas mayores, consentimiento informado, grupo vulnerable, cláusula auto ejecutable

\begin{abstract}
This paper analyzes the standards of informed consent of the elderly people in health. Firstly, we study the need for specialization in the protection of elderly people rights. Secondly, the innovation introduced by the Inter-American Convention for the Protection of the Human Rights of Older Persons with respect to informed consent and the development of this right in comparative law and case law. Thirdly, the obligations for Chile established by the InterAmerican Court of Human Rights in the Poblete Vilches v. Chile and self-execution of the Convention are examined.
\end{abstract}

${ }^{1}$ Candidato a Doctor en Derecho Universidad de Talca-Chile. Magíster en Políticas Públicas, Facultad Latinoamericana de Ciencias Sociales-Sede Ecuador. Becario de Doctorado Nacional ANID. Concepción, Chile, contacto: ecalahorrano@utalca.cl. Orcid: https://orcid.org/0000-0003-0408-5737. 
Keywords: Elderly Persons, Informed Consent, vulnerable people, Self-executing clause

\section{Introducción}

En Chile, según cifras de Censo 1992, un 6,6\% de las personas tenía 65 y más años, mientras que el Censo 2017 reveló que este grupo etario representó el 11,4\%. Los adultos mayores (60 años y más), por su parte, superan las 2 millones ochocientas mil personas, equivalente al 16,2\% de las personas efectivamente censadas ${ }^{2}$. Por lo señalado, este grupo es relevante en materia de políticas públicas y sigue en crecimiento.

La Convención Interamericana para la Protección de los Derechos de las Personas Mayores (CIDHPM $)^{3}$ se constituye como un hito en la materia al ser el primer Tratado Internacional de Derechos Humanos que implementa normativa específica respecto a los derechos de este grupo. Este instrumento genera varios desafíos para afrontar el envejecimiento de la sociedad, uno de los ejes principales se refiere al derecho de la persona mayor a emitir su consentimiento informado en el ámbito de salud ${ }^{4}$.

La Ley 20.584 que regula los derechos y deberes de las personas en relación con acciones vinculadas a su atención de salud en Chile brinda un panorama básico sobre el consentimiento libre e informado en el ámbito de salud; adicionalmente, la condena a Chile por parte de la Corte Interamericana de Derechos Humanos en el Caso Poblete Vilches vs. Chile y la imposición en esta de medidas positivas para la garantía del derecho de las personas mayores a emitir consentimiento libre e informado concuerdan con las necesidades materiales contenidas en el informe de análisis de brechas para el cumplimiento de la Convención elaborado por el Servicio Nacional del Adulto Mayor (SENAMA) ${ }^{5}$. Este panorama fáctico y normativo sobre los derechos de las personas mayores lleva a la reflexión respecto de la auto ejecutabilidad de la cláusula relativa al consentimiento informado contenida en la Convención.

La hipótesis del presente trabajo es que el derecho al consentimiento informado de la persona mayor en el ámbito de salud pertenece a la categoría de los derechos fundamentales, por lo que su contenido se ha enriquecido con la ratificación de la Convención Interamericana para la Protección de los Derechos de las Personas Mayores, por cuanto la cláusula que lo contiene es

\footnotetext{
${ }^{2}$ Instituto Nacional De Estadísticas (2018), p. 7

${ }^{3}$ La Convención Interamericana para la Protección de los Derechos de las Personas Mayores fue adoptada el 15 de junio de 2015 , entró en vigor el 11 de enero de 2017 y fue ratificada por Chile el 15 de agosto de 2017.

${ }^{4}$ Davobe (2018), p. 66.

${ }^{5}$ Servicio Nacional del Adulto Mayor (2018), p. 65.
} 
auto ejecutable. Por otro lado, las medidas que contienen deberes positivos para Chile en la sentencia del caso Poblete Vilches pueden implementarse mediante políticas públicas y normas infra legales.

Se iniciará el presente trabajo argumentando respecto a la necesidad de protección especial a las personas mayores; posteriormente se identificarán los estándares del derecho al consentimiento informado en salud de las personas mayores en el Derecho Internacional de los Derechos Humanos. Finalmente se sustentará la capacidad de auto ejecutabilidad de la cláusula de la CIDHPM referente al derecho al consentimiento informado de las personas mayores en el ámbito de salud; y la posibilidad de directa e inmediata.

\section{El fundamento de la especificación de los derechos de las personas mayores}

La manifestación de los derechos humanos en las políticas públicas requiere la protección de aquellos grupos de personas más vulnerables ${ }^{6}$, entendidas como tales aquellas que sufren maltrato estructural, es decir, multidimensional desde las estructuras de la sociedad, situación que les priva de la posibilidad de ejercer sus derechos ${ }^{7}$.

La vulnerabilidad se construye socialmente y el recorrido parte desde el rechazo por la diferencia, lo que convierte a un grupo determinado en invisible o hasta indeseado por la sociedad. En el caso de las personas mayores el criterio etario es el que determina una aparente pérdida o limitación de la autonomía. El efecto es el despojo de la autonomía, el cual se acrecienta mientras la edad avanza8.

Los derechos de la vejez son fruto del proceso de especificación y requerimiento de normas de discriminación positiva9.

La dignidad humana es el centro y fundamento de los derechos y sus garantías ${ }^{10}$. Se reconoce que la estructura social y los estereotipos impiden que todos los seres humanos tengan iguales oportunidades de ejercicio de derechos y se identifican criterios sospechosos de discriminación que deben ser equilibrados mediante medidas de atención prioritaria. Como señala Prieto

${ }^{6}$ Abramovich (2006).

${ }^{7}$ Riveros et.al. (2017), p. 164.

${ }^{8}$ Comisión Económica para América Latina y el Caribe (2018), p. 88-89.

${ }^{9}$ Dabove (2016), pp. 45-49.

${ }^{10}$ Nogueira (2015), pp. 302-306 
Sanchís, el asunto primordial consiste en determinar cuándo las diferencias de hecho provocan desigualdades jurídicas; $\mathrm{y}$, si aquellas tienen la entidad suficiente para justificar un trato distinto 11.

La justificación de la especificidad de los derechos de la vejez radica en la necesidad de exclusión de toda discriminación arbitraria, por lo que las convencionalidades sociales pasan a ser ampliamente revisadas para erradicar prejuicios y estereotipos ${ }^{12}$.

La gravedad y urgencia de atender la discriminación por edad a las personas mayores radica en que se produce, primordialmente, en el entorno familiar o de cuidado. La relación de confianza, cuidado, convivencia o dependencia constituye el punto clave para calificar una acción u omisión dañosa a una persona mayor como maltrato; el síndrome de burn out ${ }^{13}$ de los cuidadores y la invisibilización de sus funciones son elementos que caracterizan posibles situaciones futuras de maltrato.

Los factores de riesgo son múltiples ${ }^{14}$; sin embargo, la discapacidad, lentitud o limitaciones funcionales, inexperiencia de los cuidadores e historial de violencia son los más frecuentes ${ }^{15}$.

La Encuesta de Calidad de Vida en la Vejez realizada por el Servicio Nacional del Adulto Mayor mide el índice de calidad de vida que muestra el bienestar de la persona mayor en cuatro dimensiones como son condiciones físicas, materiales, relaciones afectivas y bienestar subjetivo $^{16}$. El índice de calidad de vida para las personas mayores en Chile ha mejorado desde un 0,63 a un 0,72 desde el 2007 al 2016; sin embargo, los subíndices de bienestar subjetivo y condiciones materiales han sido los peor evaluados, llegando a un 0,61 y 0,63 , respectivamente en el $2016^{17}$.

\footnotetext{
${ }^{11}$ Prieto (1995), p. 31.

12 Díaz (2015), pp. 161-164.

${ }^{13}$ El síndrome del burnout, del quemado o de desgaste ocupacional se define en la versión 04/2019 del CIE-11 como “un síndrome conceptualizado como resultado del estrés crónico en el lugar de trabajo que no se ha manejado con éxito. Se caracteriza por tres dimensiones: 1) sentimientos de falta de energía o agotamiento; 2) aumento de la distancia mental con respecto al trabajo, o sentimientos negativos o cínicos con respecto al trabajo; y 3) eficacia profesional reducida", disponible en: https://icd.who.int/browse11/l-m/es. Fecha de consulta: 01/07/2020.

14Martínez-Moreno y Bermúdez-Pérez (2016), p. 148.

${ }^{15}$ Lathrop (2009), p. 85-90.

${ }^{16}$ En la Encuesta en la Calidad de Vida en la Vejez las condiciones físicas permiten medir la autopercepción sobre la salud y las capacidades funcionales; las condiciones materiales se refieren a ingresos económicos y satisfacción de necesidades; por su parte, el subíndice de relaciones afectivas se refiere a la calidad de las relaciones familiares; y, el bienestar subjetivo a la satisfacción con la vida.

${ }^{17}$ Centro UC de Estudios de la Vejez y Envejecimiento de la Pontificia Universidad Católica de Chile y Caja los Andes (2017), pp. 1019.
} 
Lo señalado permite concluir que, en Chile, el bienestar socioeconómico de las personas mayores requiere atención especializada. El aislamiento de la persona mayor por erosión de su estructura familiar afecta su autoestima y la alimentación de estereotipos negativos como la falta de proyectos individuales ${ }^{18}$. Los estudios señalados demuestran que los problemas económicos y la preocupación por la pérdida de la autonomía son las causas principales de sentimientos depresivos en las personas mayores en Chile $^{19}$.

Como se desprende de los datos presentados, los problemas económicos y la pérdida de la autonomía son los principales problemas de las personas mayores en Chile, el desafío radica en que el derecho al consentimiento informado sea claramente entendido por los establecimientos de salud pública, altamente utilizados por este grupo etario, lo cual requiere una indagación integral del contexto del paciente mayor.

Según la Quinta Encuesta Nacional de Inclusión y Exclusión Social de las Personas Mayores en Chile, el 68\% de los encuestados respondió que las personas mayores no son auto-valentes; por otro lado, el 70\% de la muestra contestó que el país se encuentra poco o nada preparado para el envejecimiento poblacional; aunque, el 57\% señala que el principal responsable por el bienestar de las personas mayores es el gobierno. Tan solo el $44 \%$ de los encuestados estaría dispuesto a contribuir con un impuesto específico para garantizar los servicios que requieren las personas mayores.

Estos datos reflejan un prejuicio latente en la sociedad chilena respecto a la capacidad de las personas mayores y atribución de esta responsabilidad al gobierno ${ }^{20}$. Lo señalado exige focalización de las políticas de salud, especialmente en evitar la pérdida de funcionalidad o recuperarla. Estos objetivos son relevantes si se tiene en cuenta la mayor presencia de enfermedades crónicas en la vejez ${ }^{21}$.

Según datos de la II Estudio Nacional de la Discapacidad el 38,3\% de las personas mayores de 60 años en Chile tienen discapacidad y el 12,8\% de persones mayores de 60 años con discapacidad tienen dependencia funcional severa ${ }^{22}$.

\footnotetext{
${ }^{18}$ Riveros et.al (2017), p. 166.

${ }^{19}$ Centro UC de Estudios de la Vejez y Envejecimiento de la Pontificia Universidad Católica de Chile y Caja los Andes (2017), pp. 22-

23.

${ }^{20}$ Servicio Nacional del Adulto Mayor (2018), pp. 24 y ss.

${ }^{21}$ Peláez y Ferrer (2001), pp. 149-151.

${ }^{22}$ Servicio Nacional de la Discapacidad (2017), p.8.
} 
Un considerable porcentaje de la población mayor de 60 años en Chile tiene discapacidad y dependencia funcional, mayormente causada por enfermedades crónicas, por lo que recurrirán reiterativamente al sistema de salud público o privado.

El consentimiento informado del paciente es requisito fundamental para la legitimidad de la intervención médica, su omisión o cumplimiento imperfecto generan responsabilidad por omisión de una obligación legal23. La persona mayor es especialmente vulnerable respecto a la capacidad de comprensión de la información, por lo que se requieren esfuerzos para su obtención y como ultima ratio, el consentimiento por representación ${ }^{24}$.

\section{Elementos y contenido del derecho al consentimiento informado de las personas mayores en el ámbito de salud}

El acervo normativo que sirvió de antecedente a la Convención está conformado principalmente por documentos del denominado soft law ${ }^{25}$; por lo que el primer Tratado Internacional que aborda la protección de los derechos de las personas mayores, de manera específica e individualizada, es la CIDHPM ${ }^{26}$.

En 1982 se estableció el Plan de Acción Internacional de Viena sobre el Envejecimiento, primer documento que aborda la temática ${ }^{27}$. En 1991 se suscriben los Principios de Naciones Unidas en favor de las Personas de Edad ${ }^{28}$. En 1992 se conmemoró una década del Plan de Acción de Viena mediante la Proclamación del Envejecimiento en el que se instaría a los estados a implementar políticas públicas por los derechos de las personas mayores ${ }^{29}$.

La Observación General № 6 del Comité de Naciones Unidas de Derechos Económicos Sociales y Culturales emitida en 1995 evidencia que ni en el Pacto ni en la Declaración Universal de Derechos Humanos se hace explícitamente referencia a la edad como criterio sospechoso de discriminación y señala que esta omisión se explica probablemente por el hecho de que dichos

\footnotetext{
${ }^{23}$ López (2018), pp. 115-147; Pizarro (2017), pp. 40-56; Galán (2006), pp. 41-424.

${ }^{24}$ Piers et al. (2013), pp. 323-329.

${ }^{25}$ Spanier et al. (2018), pp. 55-69.

${ }^{26}$ Davobe (2016), pp. 49-50.

${ }^{27}$ Plan de Acción Internacional de Viena, 26 de julio a 6 de agosto de 1982, p. 25. El considerando 61 y la Recomendación 8 se refieren al consentimiento de las personas mayores en el ámbito de salud.

${ }^{28}$ Principios de las Naciones Unidas en favor de las Personas de Edad, 16 de diciembre de 1991. En el principio de cuidado se señala:

"las personas de edad deberán tener acceso a servicios sociales y jurídicos que les aseguren mayores niveles de autonomía, protección y cuidado".

${ }^{29}$ Proclamación del Envejecimiento, 42. Sesión Plenaria, 16 de octubre de 1992.
} 
instrumentos fueron adoptados en tiempos en que el problema del envejecimiento de la población no era tan urgente 30 .

Siete años después, el Plan de Acción Mundial sobre el Envejecimiento priorizó el fomento de la salud y bienestar en la vejez; resaltando la importancia del acceso universal y equitativo, especialmente a la atención primaria, cuidados de largo plazo; y, salud mental. El enfoque se refiere principalmente a la implementación de medidas para lograr la salud integral en las personas de edad 31 .

La Declaración de Brasilia adoptada en la Segunda Conferencia Regional Intergubernamental sobre Envejecimiento en América Latina y el Caribe se refirió al derecho de las personas mayores a cuidados paliativos y una atención médica integral y sensible ${ }^{32}$; así como, la necesidad de un mayor impulso a la investigación geriátrica y la adopción de una Convención de Naciones Unidas sobre la materia ${ }^{33}$. Esta Declaración introduce una importante modificación en la concepción de la persona mayor, desde el paradigma asistencial, hacia el de sujeto de derechos ${ }^{34}$. Respecto al ámbito específico de la salud, se concentra especialmente en el acceso, pero no existe referencia expresa a la autodeterminación ni al derecho del consentimiento informado.

El antecedente directo de la CIDHPM sería la Carta de San José sobre los derechos de las personas mayores de América Latina y el Caribe, generada por la Comisión Económica para América Latina (CEPAL) y adoptada en la tercera Conferencia Regional intergubernamental sobre envejecimiento en América Latina y el Caribe celebrada del 8 al 11 de mayo de 2012. En este documento se reafirma el compromiso realizado en la Declaración de Brasilia respecto a las personas mayores y sus derechos y se insta al Grupo de Trabajo de composición abierta sobre el envejecimiento establecido por la Asamblea General de las Naciones Unidas a adoptar una Convención Internacional en la materia. Igualmente, frente al derecho a la salud, se estableció expresamente el compromiso respecto de asegurar el derecho a un consentimiento previo, libre e informado para toda intervención médica, con independencia de la edad, condición de salud y tratamiento previsto, para favorecer la autonomía de las personas mayores; así como, el acceso a cuidados paliativos y el derecho a una muerte digna y sin dolor.

\footnotetext{
30 Observación General № 6 del Comité de Naciones Unidas de Derechos Económicos, Sociales y Culturales, E/1996/22, c. 11 .

${ }^{31}$ Informe de la Segunda Asamblea Mundial sobre el Envejecimiento, 8-12 de abril de 2002, pp. 20-25.

32 Declaración de Brasilia, diciembre de 2007, c. 15, p. 9

33 Declaración de Brasilia, diciembre de 2007, c. 20 y 26, p. 9 y 10.

${ }^{34}$ Abramovich (2006); pp. 35-50; Broberg y Sano (2018); pp. 675-676.
} 
Así las cosas, en el preámbulo de la CIDHPM se abarca expresamente el reconocimiento de la persona mayor en iguales derechos humanos y libertades fundamentales; sin embargo, se señala que la vejez puede convertirse en un criterio sospechoso de discriminación, por lo que resalta el hecho de que la persona mayor, a medida que envejece, debe seguir disfrutando de una vida plena, independiente y autónoma.

El consentimiento informado se ha consagrado como derecho de las personas y recabarlo como obligación legal de los profesionales de la salud en un buen número de legislaciones ${ }^{35}$.

Para que el consentimiento de la persona sea libre e informado se requiere previamente que el profesional de salud proporcione la información necesaria al paciente. Se ha señalado además por la doctrina que la mera transmisión de información no es suficiente; se requiere que el facultativo emita una opinión expresa sobre lo que, bajo su experticia profesional, es más favorable para el paciente ${ }^{36}$.

En el contexto europeo se emitió el Convenio Europeo para la Protección de los Derechos Humanos y la Dignidad del Ser Humano con respecto a las aplicaciones de la Biología y la Medicina (en adelante Convenio de Oviedo). El artículo 5 de esta norma establece que el consentimiento es requisito obligatorio de toda intervención médica; por lo tanto, todo acto médico que carezca del mismo es ilegítimo. Las excepciones a esta regla se desarrollan en los artículos 6 y 8 y se configuran en el consentimiento por representación en los casos en que el paciente no sea capaz de emitir su consentimiento; y, en el principio de beneficencia y atención ante una urgencia, cuando no se pueda acudir a un representante para recabar el consentimiento. Esta normativa consagra además el deber de informar del médico de manera adecuada, completa y oportuna sobre los riesgos y consecuencias de la intervención.

\footnotetext{
35 A modo de ejemplo se pueden señalar la §630d del BGB que forma parte del contrato de tratamiento incorporada mediante la promulgación de la Ley de Derechos de los Pacientes de 20 de febrero de 2013. Esta misma norma incorporó un procedimiento especial para la determinación de la responsabilidad médica Prütting (2017), pp. 265-274.

Otras legislaciones que han regulado directamente el derecho del paciente, aunque, sin acoger el modelo del contrato de tratamiento, son la Ley 41/2002 en España que en el artículo 8 que establece que toda actuación en el ámbito de la salud de un paciente necesita el consentimiento libre y voluntario del afectado; la Ley italiana 219/2018 de 16 de enero de 2018, que regula en su artículo 4 que cualquier persona mayor de edad capaz de entender suscribirla respecto a autorización o rechazo de tratamientos, pruebas diagnósticas o cualquier tipo de intervención médica, para el caso en que en un futuro no pueda manifestar su voluntad. En Francia, el artículo L 1111-4 introducido al Código de la Salud Pública por la Ley 2002-303 regula los derechos del paciente. En la región latinoamericana resulta paradigmático la inclusión detallada de los requisitos del consentimiento informado en salud en el artículo 59 del Código Civil y Comercial de la Nación Argentina; en Uruguay aparece en el artículo 11 de la Ley 18.335 de 26 de agosto de 2008 e igualmente sucede en Colombia con el artículo 10c de la Ley Estatutaria 1751 de 16 de febrero de 2015; finalmente, este derecho está expresamente consagrado en el artículo 15 del Código Civil brasileño de 2002.

En Chile es la Ley 20.584, que regula los derechos y los deberes que tienen las personas en relación con acciones vinculadas a su atención en salud, desarrolla la forma y contenido del consentimiento informado en el ámbito de salud.

${ }^{36}$ López (2018), pp. 112-117.
} 
Ya en el ámbito latinoamericano, la CIDHPM en su artículo 11 regula ampliamente el derecho de la persona mayor a consentir libremente y de manera informada en el ámbito de salud. Esta norma puede analizarse, en primer lugar, desde las características del derecho al consentimiento informado de la persona mayor; luego, el deber de informar del profesional de la salud y el contenido de la información.

\subsection{CARACTERÍSTICAS DEL DERECHO}

La CIDHPM en su artículo 11 señala que el derecho de la persona mayor al consentimiento informado en el ámbito de salud es irrenunciable, su negación constituye una forma de vulneración de los derechos humanos de la persona mayor.

La irrenunciabilidad se refiere a la indisponibilidad del derecho y su relación con la dignidad humana.

Esta característica es propia del derecho natural que señala que los derechos fundamentales son previos al derecho positivo y emanados directamente desde la dignidad humana, razón por la cual no es posible su renuncia; sin embargo, a nivel práctico, se pueden establecer circunstancias específicas en el que puede renunciarse legítimamente a su ejercicio, aunque se conserve en todo momento la titularidad ${ }^{37}$.

La renunciabilidad del derecho al consentimiento informado puede manifestarse en el requerimiento del paciente de no obtener información sobre el tratamiento, intervención o investigación a aplicársele; sin embargo, la manifestación de este deseo debe ser expresa. Además de la mencionada, las únicas excepciones admisibles para que el médico decida no transmitir la información necesaria al paciente o sus representantes son el beneficio terapéutico y la urgencia vital. Por lo mencionado, a pesar de que sea previsto en algunas legislaciones, resulta discutible recurrir a una figura como el consentimiento informado hipotético para justificar la falta de divulgación de la información por parte del tratante en situaciones distintas a las excepciones mencionadas ${ }^{38}$.

Al prescribir el artículo 11 de la Convención que la negación a una persona mayor de la posibilidad de que emita consentimiento informado en el ámbito de salud vulnera los derechos humanos se verifica una intención concreta de proporcionar a este derecho el carácter de

\footnotetext{
${ }^{37}$ Laporta (1987), p. 44.

${ }^{38}$ Pizarro (2015), p. 113-119.
} 
fundamental. Este objetivo está ligado a un aspecto histórico, por cuanto el derecho de autodeterminación del paciente surge como reacción al encarnizamiento terapéutico y a la experimentación humana no consentida, ambas consideradas prácticas aborrecibles ${ }^{39}$; pero además, por un sustento moral ligado directamente al concepto de dignidad humana ${ }^{40}$.

La Convención reitera a la dignidad como fundamento de los derechos de las personas mayores en su mismo preámbulo, luego en el artículo 3c sobre los principios rectores, el artículo 6 referido a la vida con dignidad en la vejez, el artículo 16 que regula el derecho a la privacidad e intimidad; por lo tanto, queda confirmada la intención de consolidar a las personas mayores como sujetos de derechos, pero además la obligación del estado y la sociedad de permitir el ejercicio de los mismos; sin olvidar que es un grupo vulnerable que requiere protección específica $^{41}$.

Dilucidado entonces que el derecho de las personas mayores a emitir consentimiento libre e informado en el ámbito de salud es un derecho fundamental resta indagar sobre cuáles serían las implicaciones. Sobre esto se puede encontrar una respuesta en el razonamiento de Luigi Ferrajoli, para quien un derecho debe ser considerado como fundamental si se relaciona con la paz, el alcance de la igualdad y sobre todo la protección del más débil ${ }^{42}$. Como parte de los derechos civiles, específicamente de la libertad y autonomía; el consentimiento informado protege al paciente, como parte vulnerable en la relación clínica. En palabras de Mosset Iturraspe, el Derecho Privado contemporáneo tiene como principal objetivo alcanzar la equidad y que la misma aparezca en plena magnitud a través de sus funciones integradora e interpretativa de la buena $\mathrm{fe}^{43}$.

La contractualización de la relación entre médico y paciente ha sido adoptada en algunas legislaciones como avance frente al paternalismo en la Medicina; efectivamente, asimilar la relación entre médico y paciente como un contrato da fe de la importancia del acuerdo entre las partes y la confianza para el cumplimiento de un deber de cuidado 44 .

Como todo contrato, el consenso es uno de los elementos fundamentales para su validez jurídica; sin embargo, el consentimiento libre e informado que autoriza una intervención,

\footnotetext{
39 Parra (2014), p. 211-212.

${ }^{40}$ Habermas (2010), pp. 17-23.

${ }^{41}$ Parra (2019), pp. 331-350.

${ }^{42}$ Ferrajoli (2006), pp. 133-136.

${ }^{43}$ Mosset (2011), pp. 184-185.

${ }^{44}$ Vidal (2018), pp. 13-18.
} 
procedimiento, tratamiento o investigación médica por parte del paciente es diferente del consenso contractual, por cuanto primero tiene la categoría de derecho fundamental ${ }^{45}$.

En aquellos sistemas que no han acogido o se han distanciado de la asunción de la relación médico-paciente como un contrato, la naturaleza del derecho al consentimiento informado previo es más evidente. En el Derecho anglosajón, por ejemplo, el compromiso de la integridad física del paciente por la intervención médica sin autorización es accionado por tort of battery, por lo que nos encontramos frente a un daño a la integridad física de la persona ${ }^{46}$; igualmente, el incumplimiento del deber de información del médico se puede demandar como tort of negligence y permite la compensación por los riesgos materializados no informados según el estándar informativo del paciente ${ }^{47}$.

Podemos concluir que la vulneración del derecho del paciente a emitir su consentimiento libre e informado en el ámbito de salud atenta directamente contra su autodeterminación, característica consustancial a la dignidad humana y genera responsabilidad de indemnizar por los daños causados ${ }^{48}$.

\subsection{DEBER DE INFORMAR Y CONTENIDO DE LA INFORMACIÓN}

El deber de informar es una obligación legal del facultativo para con el paciente y requiere que la entrega de información sea completa, oportuna y adecuada. Este deber equilibra la asimetría natural en materia de información en la relación entre médico y paciente, otorga contenido al consentimiento informado y permite la protección de su autodeterminación ${ }^{49}$. La importancia de la información radica en un triple fundamento 50 ; en primer lugar, constitucional, como derecho fundamental consagrado directamente en la Constitución ligado a la libertad y la autonomía; o por incorporación del Derecho Internacional de los Derechos Humanos al derecho interno. Un segundo fundamento es dogmático, bajo la observación del principio de buena fe ${ }^{51}$ y los deberes secundarios de conducta que impregnan todas las relaciones de Derecho Privado; finalmente, el legal, como sucede en Chile en la Ley 20.584.

\footnotetext{
${ }^{45}$ Parra (2019).

${ }^{46}$ Paterick et al. (2008), pp.313-319; Kessler et al (2006), pp. 240-246; Cadenas (2018), pp. 54-60.

${ }^{47}$ Turton (2018), pp. 108-134.

${ }^{48}$ Santos (2018), pp. 35-38.

${ }^{49}$ Vidal (2018), pp. 48-49.

${ }^{50}$ Lorenzetti (2016), p. 279.

${ }^{51}$ Se ha señalado en la doctrina que el fundamento del deber de informar en Chile, antes de su consagración legal expresa en la Ley 20.584, se basaba en la buena fe objetiva contractual, principio contenido en el artículo 1546 del Código Civil. Pizarro (2017), p. 42; Elorriaga y López Santa María (2017), apartado 62.
} 
La CIDHPM señala la obligación de los estados respecto de la implementación de mecanismos adecuados y eficaces para impedir los abusos y fortalecer la capacidad de la persona mayor de comprender las opciones de tratamiento existentes, sus riesgos y beneficios.

La comprensión de la información depende en gran medida de la existencia de patologías que comprometan el estado cognitivo de la persona; así como, la cercanía y vínculos entre médico y paciente. La persona mayor requiere de una preparación especial del tratante en materia comunicativa. Algunos de los problemas identificados que impiden la correcta comunicación pueden ser el daño cognitivo o la discapacidad intelectual comúnmente asociada a patologías crónicas comunes en las personas mayores; un bajo nivel de confianza entre el tratante y el paciente adulto mayor; la infantilización; el abuso del ocultamiento de información basada en un uso equivocado del concepto de beneficio terapéutico; y una sobrevaloración del consentimiento por representación en el caso de las personas mayores ${ }^{52}$.

La CIDHPM exige en su artículo 11 que la información suministrada sea adecuada; es decir, adaptada a la situación y al paciente; clara, por lo que el profesional de la salud deberá expresarla en lenguaje sencillo y sin abuso de términos técnicos que la vuelvan de difícil comprensión. La información deberá además ser oportuna, por cuanto debe permitir el ejercicio de la autodeterminación, careciendo de sentido que sea proporcionada una vez que se hayan sufrido los efectos de la intervención o tratamiento ${ }^{53}$.

La Convención va más allá y señala que la información debe prestarse sobre bases no discriminatorias, de manera accesible y comprensible según la identidad cultural, nivel educativo y necesidades de comunicación de la persona mayor. Para alcanzar el estándar requerido por el Instrumento, se debería preferir un equipo interdisciplinario y de seguimiento a largo plazo que conciba la salud de la persona mayor como un proceso ${ }^{54}$.

\footnotetext{
52 Benavides (2009), p. 65; Parra (2019).

53 Pizarro (2017), pp. 40-53; López (2018), pp. 101-112; Galán (2007), p. 404; De la Maza (2017), pp. 115-118.

${ }^{54}$ En un artículo seminal de Ezekiel y Linda Emanuel, Emanuel y Emanuel (1992), pp. 2221-2223), se describen cuatro modelos de relaciones entre médico y paciente. El primero es el paternalista en el que prima el criterio del médico sobre el mejor interés del paciente; el segundo es el informativo, en el que el médico tiene el deber de proporcionar al paciente toda la información de la manera más certera posible; $y$, este último deberá elegir entre las opciones de tratamiento informadas. En el modelo interpretativo en el cual el profesional médico tiene como objetivo interpretar los valores y preferencias del paciente y actuar en función de ellos y las intervenciones que el paciente autorice; finalmente, el modelo deliberativo se desarrolla a través de consensos. En el modelo interpretativo el rol del médico se asemeja al de un asesor; mientras que en el deliberativo al de un profesor. Se concuerda con los autores del mencionado artículo en que el modelo deliberativo es el más adecuado cuando se trata con pacientes personas mayores Una aproximación actualizada al enfoque de Advance Care Planning (planificación del cuidado en salud) se puede verificar en Sudore (2017), pp. 226-231.
} 
Por lo tanto, la Convención, respecto al deber de informar del profesional de la salud en el contexto del paciente adulto mayor, procura fortalecer la capacidad de este para comprender las consecuencias de la intervención. Este objetivo solo puede ser alcanzado mediante la implementación de una relación médico-paciente a largo plazo, que abarque la obtención de la historia de valores, metas y preferencias del paciente, consolidando una relación de confianza.

El contenido de la información a transmitirse es otro punto de discusión relevante. La Convención solo aborda someramente este tema, señalando que deben transmitirse al paciente las opciones de tratamiento, sus riesgos y beneficios.

En el ámbito europeo, el Convenio de Oviedo señala que la información a entregarse debe ser la adecuada respecto a la naturaleza y finalidad de la intervención, así como sus riesgos y consecuencias ${ }^{55}$. Una mayor precisión del contenido del deber de informar se ha desarrollado en las legislaciones nacionales ${ }^{56}$. En Chile, por ejemplo, es el artículo 10 de la Ley 20.584 el encargado de regular el deber de información del médico. Queda claro que el ejercicio del mismo es una fase anterior a recabar el consentimiento informado del paciente cuya validez depende justamente del primero.

La redacción coincide con lo establecido en otras legislaciones y es más prolija que la de la CIDHPM; sin embargo, la doctrina ha señalado la necesidad de una mayor precisión respecto a los riesgos que deben informarse, por cuanto es necesario diferenciar entre típicos y atípicos. El

\footnotetext{
${ }^{55}$ Convenio para la protección de los derechos humanos y la dignidad del ser humano con respecto a las aplicaciones de la Biología y la Medicina, 04 de abril de 1997, artículo 5.

${ }^{56}$ A manera de ejemplo se puede dar como referencia la Ley 41/2002 de España delimita el contenido del deber de informar del médico en el artículo 10.

El BW holandés regula el contenido del deber de información del médico en el artículo $7: 448$, como parte de las disposiciones del contrato de tratamiento médico.

Por su parte, el BGB alemán en la §630e señala que el facultativo debe informar todas aquellas circunstancias necesarias para el consentimiento como lo son la naturaleza, ámbito, implementación, consecuencias y riesgos de la intervención; así como, su necesidad, urgencia, idoneidad y pronóstico; y, perspectivas de éxito con respecto al diagnóstico o la terapia. Las alternativas al tratamiento también deben mencionarse en la información; y verificar si varios métodos, habitualmente indicados, pueden conducir a resultados, riesgos o posibilidades de recuperación significativamente diferentes.

El numeral 3 del artículo 1 de la Ley 219/2017 de Italia. La Ley 2002-303 en Francia o Ley Kouchner señala en su artículo L111-2 que la información debe versar respecto de la utilidad, urgencia, consecuencias y riesgos frecuentes o graves normalmente previsibles en los tratamientos, investigaciones o acciones preventivas en el ámbito médico.

En nuestra región el deber de informar del médico ha sido recogido en el Código Civil y Comercial de la Nación Argentina en el que se señala que el consentimiento informado es la consecuencia de la transmisión de información clara, precisa y adecuada respe cto a su estado de salud; el procedimiento propuesto, con especificación de los objetivos perseguidos; los beneficios esperados del procedimiento; los riesgos, molestias y efectos adversos previsibles; la especificación de los procedimientos alternativos y sus riesgos, beneficios y perjuicios en relación con el procedimiento propuesto; las consecuencias previsibles de la no realización del procedimiento propuesto o de los alternativos especificados. Este deber también aparece detallado en los artículos 11 y 18 de la Ley 18.335 de Uruguay y el artículo 10d de la Ley Estatutaria 1751 de Colombia regula el deber de informar del profesional de salud, adicionalmente, el artículo 11 señala expresamente como paciente vulnerable a la persona mayor.
} 
problema radica en que los segundos pueden ser infrecuentes estadísticamente, pero de una gravedad tal que influyan en la magnitud del daño ${ }^{57}$.

Respecto al contenido del deber de informar del profesional médico cuando el paciente es una persona mayor, podemos señalar que el artículo 10 de la Ley 20.584 obliga a un deber genérico de transmitir información respecto del estado de su salud, del posible diagnóstico de su enfermedad, las alternativas de tratamiento disponibles para su recuperación y los riesgos que ello pueda representar; así como, del pronóstico esperado, y del proceso previsible del postoperatorio, cuando procediere, de acuerdo con su edad y condición personal o emocional.

El segundo inciso de esta norma es el de mayor discusión respecto a la razonabilidad de su aplicación por cuanto exige que, si a juicio del médico tratante, la condición de la persona no le permite recibir o comprender la información, esta será entregada a su representante o quien ejerce el cuidado. La responsabilidad del médico tratante es directa, al ser este quien decide si opera o no el consentimiento por representación en una situación de pérdida de la capacidad transitoria o permanente del paciente, lo que es frecuente en las personas mayores.

En este punto nos encontramos ante un terreno espinoso en el que la única posibilidad de conseguir soluciones adecuadas es evaluar la capacidad en cada caso específico según la gravedad de la intervención, el beneficio terapéutico de entregar la información y la racionalidad de las decisiones del paciente.

El derecho a rechazar un tratamiento o intervención médica como freno al encarnizamiento terapéutico y protección a la dignidad de la persona frente a procedimientos como la alimentación e hidratación artificial sin fin terapéutico, nos permite colegir que el derecho a la autodeterminación puede incluso prevalecer, aunque el paciente tome decisiones irracionales desde el punto de vista terapéutico ${ }^{58}$.

Ante la ductilidad de los escenarios que puedan presentarse es fundamental recordar que el profesional médico no puede impedir que las personas capaces puedan decidir sobre su propio tratamiento; $y$, en segundo, debe proteger a personas que han perdido la capacidad de los efectos perniciosos de una mala decisión ${ }^{59}$.

\footnotetext{
${ }^{57}$ Pizarro (2017), pp. 46-53.

58 Zúñiga (2013), p. 123.

${ }^{59}$ Parra (2019).
} 
La jurisprudencia en Chile, aunque escasa, ha comenzado una labor cada vez más exigente de construir la forma y contenido del deber de informar.

La Corte Suprema en sentencia Rol 89.635-2016 analiza el contenido del deber de informar en casos de urgencia; así como el de derivar el caso a un profesional especializado ${ }^{60}$. Por su parte, respecto al deber de informar como requisito del consentimiento informado y la ineficacia de la suscripción del formulario del consentimiento informado por parte del paciente, sin el diálogo y clara comunicación que permita constatar que se ha cumplido con dicha obligación, puede revisarse en el fallo de Rol 87.914-201661. Resulta interesante también la sentencia de la Corte Suprema Rol 9.481-2016 en la que se acoge la tesis de condenar al Servicio de Salud del Maule por falta de información oportuna respecto a un diagnóstico de VIH que no permitió el adecuado tratamiento, concluyendo con la muerte prematura del paciente. En este fallo, se acoge como consecuencia de la falta de información una pérdida de la oportunidad del paciente ${ }^{62}$.

Estos fallos se suman a otros como la Sentencia Rol 5.849-2009 que estableció un primer contenido al deber de información del médico antes de la promulgación de la Ley 20.584, la sentencia Rol 5.396-2014 que complementa ese desarrollo; y, la sentencia Rol 21.373-2015 que incursiona respecto a la evaluación de los riesgos no informados materializados ${ }^{63}$.

Como se puede observar, la jurisprudencia ha intensificado el tratamiento del consentimiento informado especialmente en los últimos diez años con un mayor impulso desde la promulgación de la Ley 20.584.

\section{Estándares interamericanos del derecho al consentimiento informado de las personas mayores en salud: el caso Poblete Vilches vs. Chile}

El fallo trata sobre el deber estatal de asegurar el acceso de las personas a servicios esenciales de salud, asegurando una prestación médica de calidad y eficaz; así como, de impulsar el mejoramiento de las condiciones de salud de la población. Por otro lado, la Corte Interamericana se pronuncia por primera vez respecto a los derechos específicos de las personas mayores como

\footnotetext{
${ }^{60}$ Corte Suprema, Rol N ${ }^{\circ} 89.635-2016,29$ de septiembre de 2017.

${ }^{61}$ Corte Suprema, Rol № 87.914-2016, 01 de junio de 2017.

${ }^{62}$ Corte Suprema, Rol № 9.481-2016, 22 de septiembre de 2016.

${ }^{63}$ Corte Suprema, Rol № 21.373-2015, 20 de marzo de 2016.
} 
grupo en situación de vulnerabilidad y la necesidad de respeto y garantía reforzada de su derecho a la salud 64 .

Los hechos del caso describen una serie de sucesos que contravienen los derechos del paciente; es así que, el señor Poblete Vilches ingresó al Hospital Sótero del Río el 17 de enero de 2001 por insuficiencia respiratoria grave. Permaneció hasta el 22 de enero de 2001 en Unidad de Cuidados Intensivos Médica, para luego ser trasladado a una UCI quirúrgica. El 26 de enero de 2001 fue operado sin el consentimiento informado suyo o de sus familiares; a pesar de que estos habían advertido sobre el padecimiento de diabetes del paciente. El 2 de febrero de 2001 fue dado de alta prematuramente con un evidente mal estado de salud, los familiares tuvieron que trasladarlo a su casa en ambulancia privada ante la carencia de transporte del hospital. Debido al mal estado de salud fue reingresado al Hospital el 5 de febrero de 2001 requiriendo el apoyo de un ventilador mecánico que no se encontraba disponible en la UCI Quirúrgica donde fue internado, sino en la médica. Según lo declarado por los familiares afectados, la Dirección del Hospital no habría autorizado el uso del ventilador disponible. El 7 de febrero de 2001 el señor Poblete Vilches falleció debido a un shock séptico y una bronconeumonía bilateral 65 .

La Corte realiza el análisis respecto a la vulneración al derecho a la salud, la vida e integridad personal y el consentimiento informado y acceso a la información en materia de salud ${ }^{66}$.

Se establecen estándares obligatorios de cumplimiento del derecho a la salud como son la calidad, accesibilidad, disponibilidad, aceptabilidad. Dentro del criterio de accesibilidad se señala que deben erradicarse las dificultades de acceso por criterios sospechosos de discriminación, enunciados en el artículo 1.1. de la Convención Americana, entre los cuales la edad también debe ser tomada en cuenta, por cuanto el listado es ejemplificativo y no taxativo ${ }^{67}$.

La Corte establece además que las personas mayores merecen una protección reforzada como sujetos de derecho de mayor vulnerabilidad, lo cual ya había sido consagrado en las Observaciones Generales 6 y 14 del Comité de Derechos Económicos, Sociales y Culturales.

Respecto al derecho a la vida y la integridad personal, la Corte, fundamentada en el caso Lopes de Sousa Fernández vs. Portugal, resuelto por el Tribunal Europeo de Derechos Humanos,

\footnotetext{
${ }^{64}$ Corte Interamericana de Derechos Humanos, 08 de marzo de 2018, Caso Poblete Vilches vs. Chile, Serie C-349. p. 3.

65 Para un mayor detalle de los hechos consultar Corte Interamericana de Derechos Humanos, 08 de marzo de 2018 , Caso Poblete Vilches vs. Chile, Serie C-349 pp. 13-19.

${ }^{66}$ Corte Interamericana de Derechos Humanos, 08 de marzo de 2018, Caso Poblete Vilches vs. Chile, Serie C-349 p. 30.

${ }^{67}$ Corte Interamericana de Derechos Humanos, 08 de marzo de 2018, Caso Poblete Vilches vs. Chile, Serie C-349 p. 40 . Este criterio es consonante con la doctrina. Comisión Económica para América Latina y el Caribe p. 88-94; Figueroa (2013), pp.12-14.
} 
recogió tres criterios para determinar la existencia de responsabilidad del Estado en casos de muerte de una persona en el contexto médico, que son: denegación de la asistencia de salud con ante una previsibilidad de riesgo a la vida del paciente; acreditación de una negligencia médica grave; existencia de nexo causal entre el acto acreditado y el daño sufrido por el paciente68.

Se determinó finalmente que Chile violó el derecho a la vida, por cuanto negó al señor Poblete Vilches un tratamiento médico de urgencia, a sabiendas de que la falta de tratamiento ponía en peligro su vida y en total desconsideración hacia su situación de adulto mayor.

Respecto al tema central de este trabajo, el consentimiento informado; la Corte analiza, en primer lugar, la normativa vigente al momento de ocurrir los hechos, de la cual se desprende claramente la obligación de informar sobre el diagnóstico, el pronóstico, las medidas terapéuticas o médico quirúrgicas que se implementaría, el objetivo, método, duración probable y riesgos del tratamiento a aplicarse; las alternativas de tratamiento, indicando expresamente las menos intrusivas; $y$, las consecuencias del mismo ${ }^{69}$.

La Corte señala que en el caso no se obtuvo el consentimiento informado por representación de parte de los familiares, ante la primera intervención quirúrgica; $\mathrm{y}$, posteriormente del propio señor Poblete Vilches, a medida que había recuperado progresivamente su capacidad para decidir.

La Corte entiende que la necesidad de obtención del consentimiento informado protege no solo el derecho del paciente a decidir libremente si desea someterse o no a un acto médico, sino que es un mecanismo fundamental para lograr el respeto y garantía de distintos derechos humanos reconocidos por la Convención Americana, como lo son la dignidad, libertad personal, integridad personal, incluida la atención a la salud, la vida privada y familiar. De este modo, la existencia de una conexión entre el consentimiento informado con la autonomía personal y la libertad de tomar decisiones sobre el propio cuerpo y la salud exige, por un lado, que el Estado asegure y respete decisiones y elecciones hechas de forma libre y responsable y, por otro, que se garantice el acceso a la información relevante para que las personas estén en condiciones de

\footnotetext{
${ }^{68}$ Corte Interamericana de Derechos Humanos, 08 de marzo de 2018, Caso Poblete Vilches vs. Chile, Serie C-349 p. 48

${ }^{69}$ Para esta definición del contenido de la información exigible al momento de producirse los hechos la Corte se refirió al Caso I.V. vs. Bolivia; así como, el Decreto Supremo № 42 de 1986 que aprobó el Reglamento Orgánico de los Servicios de Salud; y, las Normas y Documentos de la Ética Médica del Colegio Médico de Chile.
} 
tomar decisiones informadas sobre el curso de acción respecto a su cuerpo y salud de acuerdo a su propio plan de existencia ${ }^{70}$.

La Corte concluye que el estado de Chile vulneró el derecho del señor Poblete Vilches y sus familiares a emitir su consentimiento informado previo a intervenciones médicas, el derecho de acceso a la información de los familiares, por cuanto, en el segundo ingreso al Hospital no se les brindó información clara y accesible sobre el estado de salud del paciente ${ }^{71}$.

Dentro de las medidas de reparación señaladas por la Corte Interamericana se requiere a Chile la reapertura de las investigaciones referentes al caso; capacitación especializada al personal médico del Hospital Sótero del Río respecto a la atención y los derechos de las personas mayores. Adicionalmente se dispone incidencia geriátrica en la salud y medidas específicas en favor de las personas mayores, a través del fortalecimiento del Instituto Nacional de Geriatría y su presencia en la red hospitalaria; la emisión de una cartilla que contenga los derechos de las personas mayores; y su protección integral72.

El estado actual del cumplimiento de las medidas se remite al 16 de agosto de 2019, fecha en que venció el plazo para el pago de indemnizaciones. El estado ha cumplido solamente tres de las nueve medidas ordenadas por el Tribunal Regional73.

La sentencia fue notificada al estado de Chile el 21 de junio de 2018 y la primera Resolución correspondiente a la supervisión de cumplimiento de la misma el 14 de mayo de 2019. Este último documento señala que las medidas cumplidas son la publicación del resumen oficial de la Sentencia en el Diario Oficial y en el diario de amplia circulación nacional "El Mercurio" el 20 de diciembre de $2018^{74}$. El texto integral de la Sentencia en el sitio web oficial del Ministerio de Relaciones Exteriores; de la misma manera, se aclara respecto a los destinatarios del pago por indemnización por daño material, reingreso de costas y gastos y daño inmaterial, ante la aparición de nuevos herederos del destinatario.

\footnotetext{
${ }^{70}$ Corte Interamericana de Derechos Humanos, 08 de marzo de 2018, Caso Poblete Vilches vs. Chile, Serie C-349 p. 55.

${ }^{71}$ Corte Interamericana de Derechos Humanos, 08 de marzo de 2018, Caso Poblete Vilches vs. Chile, Serie C-349 p. 56.

72 Corte Interamericana de Derechos Humanos, 08 de marzo de 2018, Caso Poblete Vilches vs. Chile, Serie C-349 pp. 70-71.

${ }^{73}$ El Mercurio (2019) "CIDH sentencia a Chile por no otorgar derecho a la salud a adulto mayor" [Fecha de consulta: 25 de septiembre de 2019].[Disponible en: https://bit.ly/3g5MKHg].

${ }^{74}$ La publicación fue realizada en el "El Mercurio" de 20 de diciembre de 2018.
} 
Por otro lado, el procedimiento de supervisión se mantiene abierto respecto a las otras medidas ordenadas que se refieren a la reparación simbólica o de implementación de políticas públicas.

\section{Auto ejecutabilidad en el ordenamiento jurídico chileno de la cláusula de la CIDHPM sobre consentimiento informado}

La CIDHPM y la sentencia de la Corte Interamericana de Derechos Humanos en el caso Poblete Vilches y otros vs. Chile han generado obligaciones positivas para el estado, respecto a la garantía de los derechos de las personas mayores.

La comunión entre el Derecho Internacional Público y el Derecho Constitucional, a través del artículo 5 inciso segundo de la Carta Magna, ha permitido fortalecer el catálogo de derechos en el ordenamiento jurídico chileno y sus garantías ${ }^{75}$; por otro lado, la doctrina del control de convencionalidad, establecida por la jurisprudencia de la Corte Interamericana de Derechos Humanos $^{76}$ ha permitido consolidar un acervo normativo regional que desarrolla derechos denominado Corpus Iuris Interamericano.

A pesar de que el contenido de este compendio ha sido motivo de discusión por parte de la doctrina ${ }^{77}$; es indudable que, mínimamente están contenidos los Tratados Internacionales de Derechos Humanos a nivel regional y la jurisprudencia de la Corte Interamericana de Derechos Humanos que contiene la interpretación auténtica de la Convención Interamericana de Derechos Humanos en los casos concretos ${ }^{78}$.

Como se ha señalado, la Convención Interamericana para la Protección de los Derechos de las Personas Mayores incorpora en su artículo 11 el consentimiento informado y libre en el ámbito de salud como un derecho autónomo e irrenunciable, cuyo irrespeto implica una vulneración de los derechos fundamentales de la persona, según se ha sostenido en el presente trabajo. El inciso segundo de esta norma establece deberes positivos a los estados parte, a través de la

\footnotetext{
${ }^{75}$ Parte de la doctrina sostiene de la auto ejecutabilidad de los Tratados Internacionales de Derechos Humanos, a través del control de convencionalidad interno, para lo cual se recurre al principio de pacta sunt servanda, por el cual el incumplimiento directo e inmediato de los Tratados provocaría que Chile incurra en responsabilidad internacional. Nogueira (2013), pp 234-239; Aguilar y Nogueira (2016), pp. 22-23. Por otro lado, existe también la preocupación respecto a los efectos de los Tratados Internacionales y su auto ejecutabilidad. Esto por cuanto remplazan a la Ley que ha sido concebida bajo el debate democrático como se puede observar en Fuentes et al. (2018), pp. 135-140.

${ }^{76}$ Henriquez-Viñas (2016), pp. 137-143.

${ }^{77}$ Contreras (2017), pp. 145-157.

${ }^{78}$ Así lo establece el artículo 62 inciso tercero de la Convención Americana sobre Derechos Humanos.
} 
implementación de políticas que permitan garantizar a la persona mayor el fortalecimiento de su capacidad para comprender sus opciones de tratamiento, riesgos y beneficios; ante una intervención médica ${ }^{79}$.

El contraste necesario para advertir el aporte específico del artículo 11 de la CIDHPM debe ser respecto a los artículos 10,14, 15 y 16 de la Ley 20.584 que regulan el deber de informar del facultativo; así como, los requisitos y contenido del consentimiento informado.

Sobre este punto, resultan especialmente novedosos los dos últimos incisos del artículo 11 de la CIDHPM que regulan el derecho a aceptar, negarse a recibir o interrumpir tratamientos médicos o quirúrgicos; así como, el desarrollo de la emisión de voluntades anticipadas. Ambas normas se refieren a derechos fundamentales de la persona mayor al final de la vida.

Tanto la Convención como su interpretación realizada por la Corte Interamericana de Derechos Humanos en el caso Poblete Vilches vs. Chile son exigibles directamente al insertarse al ordenamiento jurídico, al menos con un rango de Ley; además de ser vinculantes por el principio de pacta sunt servanda; y, en función de lo contenido en el artículo 2 del Pacto de San José que obliga a los Estados Partes a adoptar, con arreglo a sus procedimientos constitucionales y a las disposiciones de esta Convención, las medidas legislativas o de otro carácter que fueren necesarias para hacer efectivos tales derechos y libertades. El reconocimiento expreso de la competencia de la Corte Interamericana por parte de Chile permitiría además que su razonamiento pueda ser invocado por tribunales nacionales.

Finalmente, tomando en cuenta las críticas a la expansión del efecto del control de convencionalidad del Pacto de San José80; es de gran utilidad el análisis sobre la auto ejecutabilidad de los Tratados Internacionales en Chile y el Corpus Iuris Interamericano como acervo normativo ampliado.

El Tribunal Constitucional no ha sido unívoco en su jurisprudencia respecto a la consideración de cuándo una cláusula de un Tratado Internacional es o no auto ejecutable, aunque se considera tal "cuando es normativamente autosuficiente para ingresar al

\footnotetext{
${ }^{79}$ Estos deberes no se limitan al ámbito legislativo; por cuanto ya varios países de la región cuentan con leyes que regulan los derechos de los pacientes y el consentimiento informado, como la Ley 20.584 en Chile. El verdadero desafío se genera respecto de las políticas públicas llevadas a cabo por los organismos gubernamentales que implementan programas y proyectos para las personas mayores, como la SENAMA en Chile; y, la manera en que estas puedan incidir en las prácticas de los profesionales sanitarios, pacientes y cuidadores para un consentimiento informado.

${ }^{80}$ Fuentes y Pérez (2018), pp. 136-137; Henríquez y Núñez (2016), pp. 137-139.
} 
ordenamiento jurídico y ser aplicada por el operador jurídico nacional, en especial, la judicatura ordinaria. Viceversa, no es autoejecutable cuando no es autosuficiente"81.

Al carecer la Constitución chilena de una norma que regule la jerarquía normativa de los Tratados Internacionales y sus efectos, este tema se ha tratado reiteradamente en la doctrina y tiene efectos prácticos respecto al reconocimiento y directa aplicación de derechos contenidos en Tratados pertenecientes al Derecho Internacional de los Derechos Humanos.

La falta de uniformidad en el criterio del Tribunal Constitucional de Chile sobre la materia se evidencia, por ejemplo, en la sentencia contenida en Rol 1340-0982, en la que se estableció el derecho implícito a la identidad; de la misma manera, en Rol № 2273-1283 donde se hizo un reconocimiento de Tratados Internacionales como límites al poder del estado, parámetros de interpretación constitucional e inclusive se reconoce estatuto jurídico en materia de extranjería ${ }^{84}$; sin embargo, la aplicación directa de los Tratados Internacionales no ha sido la regla por parte del Tribunal.

Por lo tanto, para el Tribunal Constitucional los Tratados Internacionales no son fuente directa de derechos fundamentales, solo son tales los derechos esenciales que emanan de la naturaleza humana que ellos contengan; por lo tanto, tampoco constituyen un parámetro de constitucionalidad o requieren anclarse a un derecho constitucional para su aplicación ${ }^{85}$.

En síntesis, la doctrina del Tribunal Constitucional sobre la auto ejecutabilidad de las cláusulas de los Tratados Internacionales señala que solo las normas que tienen estas características pueden tener un control previo de constitucionalidad; la excepción a esta regla es

cuando el no examen de constitucionalidad de una norma no autoejecutable de un tratado pueda derivar en la afectación del objeto y fin del mismo en su vigencia en el orden jurídico internacional, conforme al artículo 18 de la Convención de Viena sobre el Derecho de los Tratados, generando responsabilidad internacional del Estado ${ }^{86}$.

\footnotetext{
${ }^{81}$ Yáñez (2016), p. 232.

82 Muñoz con Muñoz (2009): Tribunal Constitucional de Chile, 04 de marzo de 2009, Rol 1340-09.

${ }_{83}$ Alerte Daniel/ Subsecretario del Interior del Ministerio del Interior y Seguridad Pública y Jefe del Departamento de Extranje ría y Migración del Ministerio del Interior y Seguridad Pública (2013): Tribunal Constitucional de Chile, 4 de julio de 2013, Rol 2273- 
Finalmente, aun auto ejecutable, una cláusula puede requerir legislación o reglamentación para surtir efectos.

El centro de la crítica a la auto ejecutabilidad de los Tratados Internacionales es el principio democrático y el inconveniente de que normas negociadas por el poder ejecutivo y que no se sometieron al debate parlamentario, puedan inclusive dejar sin efecto leyes, por aplicación del control de convencionalidad interno ${ }^{87}$.

Las materias de reserva de ley contenidas en el artículo 63 de la Constitución requerirían el debate democrático para su legitimidad; sin embargo, si se realiza una interpretación integral de esta norma constitucional con la contenida en el artículo 5 inciso segundo, se puede concluir la auto ejecutabilidad de los Tratados Internacionales que versan sobre Derechos Humanos por mandato constitucional; sin embargo, otros factores influyen en la capacidad auto ejecutiva del Tratado; como la especificidad de su texto; la necesidad de implementación de un régimen jurídico de remplazo al que es contrario al Tratado; el requerimiento de tipificación de delitos; o la atribución expresa de competencias a un órgano estatal. Todas estas materias precisan de una Ley para su desarrollo, por lo que el Tratado que prevea las mismas no es auto ejecutable88.

Por lo señalado, a nuestro criterio, la cláusula relativa al consentimiento informado en la CIDHPM no está comprendida en aquellas categorías que podrían retarle su capacidad de auto ejecutabilidad, por lo que puede ser invocada y aplicada directamente, en armonía con el contenido de los artículos 10,14, 15 y 16 de la Ley 20.584, inclusive en lo referente al derecho de rechazo al tratamiento. Lo señalado se refuerza con la garantía del derecho a la autonomía del paciente adulto mayor y su protección reforzada requerida por la Corte Interamericana de Derechos Humanos en el fallo Poblete Vilches.

Por otro lado, al ser jurisprudencia obligatoria e interpretación auténtica del artículo 26 de la Convención Americana de Derechos Humanos, las apreciaciones de la Corte, conjuntamente con las disposiciones de la Convención para la Protección de los Derechos Humanos de las Personas Mayores se insertan en el ordenamiento jurídico chileno con una serie de prescripciones obligatorias.

${ }^{87}$ Fuentes y Pérez (2018), pp. 132-135.

${ }^{88}$ Fuentes y Pérez (2018), pp. 140-151. 


\section{Conclusiones}

Las personas mayores son un grupo política y jurídicamente relevante para Chile; lo primero por el aumento progresivo y permanente de la población por sobre los sesenta y cinco años de edad ${ }^{89}$;lo segundo, porque el país ha ratificado la Convención Americana para la Protección de los Derechos Humanos de las Personas Mayores el 11 de julio de 2017, ante lo cual se incorporan al ordenamiento jurídico chileno algunas especificidades respecto a derechos de la persona mayor ${ }^{90}$

La vulnerabilidad producida por la pérdida de la capacidad justifica la especificación de derechos de la vejez a través de un régimen jurídico que proteja a las personas mayores de la discriminación por edad.

El derecho al consentimiento informado de las personas mayores es introducido expresamente en el artículo 11 de la CIDHPM y su fundamento radica en la dignidad humana; sin embargo, puede ser renunciable ante excepciones como el derecho a no ser informado, la urgencia vital o el beneficio terapéutico.

El derecho al consentimiento informado es un derecho fundamental, su vulneración afecta la dignidad y autonomía del paciente y exige del tratante la transmisión de información adecuada, oportuna y clara ante cualquier intervención. En el caso de las personas mayores, el cumplimiento de estas características exige el desarrollo de habilidades comunicativas por parte del tratante y que se preocupe por la historia de valores del paciente.

La CIDHPM introduce un contenido novedoso respecto del derecho a rechazar un tratamiento lo que requiere verificar la capacidad de auto ejecutabilidad de la cláusula relativa a consentimiento informado de las personas mayores en el ordenamiento jurídico chileno. La introducción de estándares específicos sobre el consentimiento informado de las personas mayores en el ordenamiento jurídico chileno también encuentra como fuente el fallo de la Corte Interamericana de Derechos Humanos Poblete Vilches y otros vs. Chile.

\footnotetext{
${ }^{89}$ Según la Síntesis de Resultados del censo 2017, el porcentaje de personas mayores de 65 años en Chile es del 11,4\%, casi el d oble del 6,6\% existente en el año 1992. La población entre $0-14$ años se ha reducido de $29,4 \%$ a $20,1 \%$ en este mismo periodo; lo que demuestra que la tendencia de envejecimiento es sostenida en los últimos 25 años.

${ }^{90}$ Se incorporan en esta Convención derechos novedosos como la autonomía e independencia; integración comunitaria; aquellos correspondientes a las personas mayores en atención a largo plazo; así como un catálogo importante de derechos sociales y culturales como a la vivienda, a la salud, a la recreación, esparcimiento y deporte.
} 
Finalmente, se considera que la cláusula de la CIDHPM, respecto al derecho del consentimiento informado de las personas mayores en materia de salud es auto ejecutable, tiene ya su desarrollo normativo en la Ley 20.584, por lo que, en el caso concreto, estas normas secundarias deben ser interpretadas en armonía con la Convención.

\section{Bibliografía citada}

Abramovich, Víctor (2006): “Una aproximación al enfoque de derechos en las estrategias y políticas de desarrollo", en: Revista CEPAL, (№ 88), pp. 35-50.

Aguilar, Gonzalo y Nogueira Alcalá, Humberto (2016): "El principio favor persona en el derecho internacional y en el derecho interno como regla de interpretación y de preferencia normativa” en Revista de Derecho Público. (№ 84. 20 de septiembre), pp. 13-43.

Benavides, Alfredo (2009): “La bioética y el adulto mayor” en Horizonte Médico, (Vol. 9, № 2), pp. 61-66.

Broberg, Morten y Sano, Hans-Otto (2018): "Strengths and weaknesses in a human rightsbased approach to international development - an analysis of a rights-based approach to development assistance based on practical experiences" en The International Journal of Human Rights (Vol. 22, № 5), pp. 664-680.

Brunet, Nicolás (2017): “Défaut d'information préalable: présomption de la souffrance morale et préjudice d'impréparation", en Médecine et Droit. (Vol. 2017, № 142), pp. 11-14.

Cadenas Osuna, Davinia (2018): “El consentimiento informado y el rechazo a la intervención o el tratamiento médico por el menor de edad tras la reforma de 2015: estudio comparado en el common law" en Anuario de Derecho Civil (№ 71); pp. 789-853.

Centro UC de Estudios de la Vejez y Envejecimiento de la Pontificia Universidad Católica de Chile y Caja los Andes (2017): “Chile y sus Mayores. 10 años de la Encuesta Calidad de Vida en la Vejez" [Disponible en: https://bit.ly/34L7ga], [Fecha de consulta: 25 de septiembre de 2019]. 
Comisión Económica para América Latina y el Caribe (2018): “Envejecimiento, personas mayores y Agenda 2030 para el Desarrollo Sostenible: perspectiva regional y de derechos humanos" (Santiago, CEPAL).

Contreras, Pablo (2017): “Notas sobre el Corpus iuris Interamericano”, en Nogueira, Humberto y Aguilar, Gonzalo coord. Control de Convencionalidad, corpus iuris y ius commune interamericano, (Santiago, Triángulo), pp. 145-157.

Dabove, María Isolina (2016): “Derechos Humanos de las Personas Mayores en la Nueva Convención Americana y Sus Implicancias Bioéticas" en Revista Latinoamericana de Bioética (Vol. 16, №1), pp. 38-59.

Dabove, María Isolina (2018): “Autonomía y vulnerabilidad en la vejez: respuestas judiciales emblemáticas" en Revista de Derecho Privado, (Vol. 34, marzo de 2018), pp. 53-85.

Díaz de Valdés, José Manuel (2015): “La igualdad constitucional: múltiple y compleja" en Revista chilena de derecho, (Vol. 42. № 1), pp. 153-187.

Emanuel, Ezekiel J. y Emanuel, Linda (1992): "Four Models of the Physician-Patient Relationship" en JAMA 267, (№ 16), pp. 2221-2226.

Ferrajoli, Luigi (2006): "Sobre los derechos fundamentales”. Cuestiones constitucionales, (№ 15, diciembre), pp. 113-136.

Figueroa, Rodolfo (2013): “Consentimiento informado en la nueva ley de derechos de los pacientes" en Revista médica de Chile (Vol. 140): pp. 1347-1351.

Fuentes, Ximena (2007): El derecho internacional y el derecho interno: definitivamente una pareja dispareja en Revista de Economía y Derecho, (Vol. 15), (n.o 4).

Fuentes, Ximena y Pérez, Diego (2018): “El efecto directo del derecho internacional en el derecho chileno" en Revista de derecho (Coquimbo), (Vol. 25. № 2, diciembre), pp. 119-156.

Galdámez, Liliana (2013): “Comentario jurisprudencial: la consulta a los pueblos indígenas en la sentencia del Tribunal Constitucional sobre ley de pesca roles números 2387-12 cpt y 2388-12 cpt, acumulados”. Estudios Constitucionales (año 11, № 1), pp. 621-632. 
Galán, Julio César (2007): Responsabilidad civil médica, 2da ed. (Cizur Menor, Navarra. Thomson-Civitas).

Habermas, Jürgen (2010): “El concepto de dignidad humana y la utopía realista de los derechos humanos". Diánoia, (Vol. 55. № 64, mayo), pp. 3-25.

Henríquez-Viñas, Miriam y Núñez, José (2016): “El control de convencionalidad: ¿hacia un no positivismo interamericano?" Iuris Tantum Revista Boliviana de Derecho. № 21 enero.

Instituto Nacional de Estadísticas (2018): “Síntesis De Resultados Censo 2017” [Disponible en: https://bit.ly/3yY8NZ4] [fecha de consulta: 27 de septiembre de 2019].

Kessler, Daniel et.al (2006): "Effects of the medical liability system in Australia, the UK, and the USA" en The Lancet. (Vol. 368, № 9531. 15 de julio), pp. 240-246.

Laporta San Miguel, Francisco (1987): “Sobre el concepto de derechos humanos” en Doxa (№ 04), pp. 23-46.

Lathrop, Fabiola (2009): "Protección Jurídica de los Adultos Mayores en Chile”, en Revista chilena de derecho, (Vol. 36, № 1, abril), pp. 77-113.

López Mesa, Marcelo (2018): “La Responsabilidad Civil Médica. Responsabilidad de sanatorios y hospitales. En el nuevo Código Civil y Comercial. Derecho Comparado" (Buenos Aires. BdeF).

Elorriaga, Fabián y López Santa María, Jorge (2017): Los Contratos. Parte General, apartado 62. Disponible en [Thomson Reuters Proview]. [Fecha de consulta: 29 de septiembre de 2019].

Lorenzetti, Ricardo (2016): Responsabilidad Civil de los Médicos. (Buenos Aires, RubinzalCulzoni Editores). Tomo I.

Mosset Iturraspe, Jorge (2011): Derecho Civil Constitucional, (Buenos Aires, Rubinzal-Culzoni).

Nogueira, Humberto (2013): "El control de convencionalidad y el diálogo interjurisdiccional entre tribunales nacionales y Corte Iberoamericana de Derechos Humanos" en Revista de Derecho Constitucional Europeo, (Año 10, № 19), pp. 221-270. 
Nogueira, Humberto (2015): “El Bloque Constitucional de Derechos en Chile, el Parámetro de control y consideraciones comparativas con Colombia y México: doctrina y jurisprudencia" en Estudios constitucionales (Vol. 13, №2), 301-50.

Parra, Darío (2014): “La evolución ético-jurídica de la responsabilidad médica" en Acta bioethica (Vol. 20, № 2, noviembre), pp. 207-213.

Parra, Darío (2019): “La autonomía de los pacientes adultos mayores en el ámbito de la salud” [Disponible en: Estudios de Derecho de Familia IV versión Thomson Reuters Proview][Fecha de consulta: 28 de septiembre de 2019].

Paterick, Timothy et. al (2008). "Medical Informed Consent: General Considerations for Physicians”, Mayo Clinic Proceedings. (Vol. 83, № 3 marzo), pp. 313-319.

Peláez, Martha y Ferrer, Marcela (2001). “Salud Pública y los Derechos Humanos de los Adultos Mayores" en Acta bioethica (Vol. 7, № 1), pp. 143-155.

Piers, Ruth et.al (2013). "Advance Care Planning in terminally ill and frail older persons" en Patient Education and Counseling. Quality of Communication from the Patient Perspective, (Vol. 90. № 3.1 de marzo), pp. 323-329.

Pizarro, Carlos (2015): “En oposición al consentimiento hipotético informado”, en: Revista de Derecho de la Pontificia Universidad Católica de Valparaíso,(Vol. 44), 2015, pp. 97-120.

Pizarro, Carlos (2017): La responsabilidad civil médica. Monografías (Santiago, Thomson Reuters).

Prieto Sanchís, Luis (1995): “Los derechos sociales y el principio de igualdad sustancial” en Revista del Centro de Estudios Constitucionales (22, septiembre-diciembre), pp. 9-57.

Prütting, Hans (2017): “Conferencia: el proceso civil por responsabilidad médica en el Derecho Alemán"; en Revista Chilena de Derecho Privado (№ 29), pp. 265-274.

Riveros, Carolina et. al. (2017): "El maltrato estructural a persona mayores en Chile y la necesidad de formular un índice multidimensional” en Revista UNIVERSUM (Vol. 32, № 2), pp. 163-176. 
Santos Morón, María José (2018): “La responsabilidad médica (en particular en la medicina voluntaria): una relectura desde el punto de vista contractual” en: In Dret (№ 1), pp. 1-57.

Servicio Nacional de la Discapacidad (2017): "II Estudio Nacional de la Discapacidad", [Disponible en: https://bit.ly/3cfoFN0] [fecha de consulta: 25 de septiembre de 2019].

Servicio Nacional del Adulto Mayor (2018): “Convención Interamericana sobre la Protección de los Derechos Humanos de las Peronas Mayores. Análisis de Brechas Legislativas y Propuestas para su Implementación en Chile.", [fecha de consulta: 20 de agosto de 2019][Disponible en: https://bit.ly/3vPi17N].

Spanier, Benny et.al (2016): "In Course of Change: Soft Law, Elder Rights, and the European Court of Human Rights", en Law \& Inequality: A Journal of Theory and Practice (Vol. 34, № 1).

Sudore, Rebecca et.al (2017): "Defining Advance Care Planning for Adults: A Consensus Definition from a Multidisciplinary Delphi Panel" en Journal of Pain and Symptom Management. (Vol. 53, № 5. 1 de mayo), pp. 821-832.

Turton, Gemma (2019): "Informed Consent to Medical Treatment Post-Montgomery: Causation and Coincidence" en Medical Law Review, (Vol. 27, № 1), pp. 108-134.

Universidad de Santiago de Chile y OTIC del Comercio, Servicios y Turismo (2016) "Mercado Laboral, adulto mayor y personas próximas a jubilar en Chile”. (Santiago, Universidad de Santiago de Chile).

Vidal Olivares, Álvaro (2018): Responsabilidad civil médica (Santiago, Chile. Cuadernos Jurídicos de la Academia Judicial, Ediciones DER).

Yánez Espinoza, Manuel (2016): “La jurisprudencia del Tribunal Constitucional chileno sobre tratados internacionales: el específico caso de las cláusulas autoejecutables y no autoejecutables. Revista Derecho del Estado. (Vol. 37), pp. 229-254.

Zúñiga Fajuri, Alejandra (2013): "La nueva ley de derechos del paciente: del modelo de la beneficencia al modelo de autonomía” en: Revista médica de Chile (Vol. 141), pp. 203-204. 


\section{Normas jurídicas citadas}

Declaración de Hong Kong, Asociación Médica Mundial sobre el Maltrato de Ancianos, 2005 [Disponible en: https://bit.ly/35tTwS0] [Fecha de consulta: 29 de septiembre de 2019]

Convención Interamericana de los Derechos Humanos de las Personas Mayores, 15 de junio de 2015, Organización de los Estados Americanos.

Declaración de Brasilia, Segunda Conferencia Regional Intergubernamental sobre Envejecimiento en América Latina y el Caribe: hacia una sociedad para todas las edades y de protección social basada en derechos, del 4 al 6 de diciembre de 2007.

Carta de San José sobre los derechos de las personas mayores de América Latina y el Caribe. Tercera Conferencia regional intergubernamental sobre envejecimiento en América Latina y el Caribe fue organizada por la CEPAL y el Gobierno de Costa Rica, 8-11 de mayo de 2012.

Ley 20.584, sobre los derechos y los deberes de las personas en las acciones vinculadas a la salud. Diario Oficial 24 de abril de 2012

Ley 18.335, sobre pacientes y usuarios del servicios de salud, Diario Oficial de 26 de agosto de 2008, República Oriental de Uruguay.

Ley 26.529, sobre los derechos de los pacientes, Boletín Oficial de 19 de noviembre de 2009, Congreso de la República Federal de Argentina.

Ley 26.994, Código Civil y Comercial de la Nación, Boletín Oficial de 08 de octubre de 2014, Congreso de la República Federal de Argentina.

Patient Self Determination Act de 1990, 03 de abril de 1990, H.R.4449 United States of America Congress.

Ley 2002-303 relative aux droits des malades et à la qualité du système de santé (relativa a los derechosde los pacientes y a la calidad del sistema de salud), de 04 de marzo de 2002. República de Francia.

Ley 41/2002, básica reguladora de la autonomía del paciente y de derechos y obligaciones en materia de información y documentación clínica, Boletín Oficial del Estado número 274 de 15 de noviembre de 2002, Jefatura de Estado, Reino de España. 
Ley 219/2018, sobre el consentimiento informado y las voluntades anticipadas, Gazzetta Ufficiale Serie Generale №12 de 16 de enero de 2018, Presidente de la República de Italia.

Bürgerliches Gesetzbuch BGB, Código Civil Alemán, Boletín del Reich, 24 de agosto de 1896. Reformado al 20 de febrero 2013.

Burgerlijk Wetboek BW, Código Civil Holandés, 01 de enero de 1992.

Bill 125, Código Civil de Quebec, 01 de enero de 1994. Parlamento de la Provincia de Quebec.

Ley 23, 18 de febrero de 1981, Diario Oficial № 35.711 de 27 de febrero de 1981, Congreso Nacional de Colombia.

Ley 1733-2014 de 8 de septiembre de 2014, Diario Oficial. Año cl. № 49268 de 8, septiembre, 2014, Congreso Nacional de Colombia.

Ley Estatutaria 1751, 16 de febrero de 2015, Diario Oficial № 49.427 de 16 de febrero de 2015, Congreso Nacional de Colombia.

Ley 10.406, Código Civil Brasileño, de 10 de enero de 2002, Diario Oficial, № 8 de 11 de enero de 2002, Congreso Nacional de Brasil.

\section{Jurisprudencia citada}

Corte Suprema: Fernando Emilio Serce Medina y otra con Servicio de Salud de O'Higgins y otros, Rol N 89.635-2016 de 29 de diciembre de 2017.

Corte Suprema: Nancy Uribe Uribe y Servicio de Salud de Reloncaví, Rol 87.914-2016 de 01 de junio de 2017.

Corte Suprema: Juan Sarabia Tolosa y otros contra Servicio de Salud del Mauley otros, Rol 9.4812016 de 22 de septiembre de 2016.

Tribunal Constitucional: Muñoz con Muñoz, Rol 1.340-09 de 04 de marzo de 2009.

Tribunal Europeo de Derechos Humanos: Lopes de Sousa Fernandes vs. Portugal, № 56080/13 de 19 de diciembre de 2017. 
Corte Suprema: P.S.M contra K.G.O, Rol 21.373-2015 de 20 de marzo de 2016, ID Vlex: 619494714.

Corte Interamericana de Derechos Humanos: Poblete Vilches vs. Chile, Caso, Serie C-349, 08 de marzo de 2018. 